\title{
NEAR-SHORE FLOATING WAVE ENERGY CONVERTERS: APPLICATIONS FOR COASTAL PROTECTION
}

\author{
Piero Ruol ${ }^{1}$, Barbara Zanuttigh ${ }^{2}$, Luca Martinelli ${ }^{2}$, Jens Peter Kofoed ${ }^{3}$ and Peter Frigaard ${ }^{3}$
}

\begin{abstract}
Aim of this note is to analyse the possible application of a Wave Energy Converter (WEC) as a combined tool to protect the coast and harvest energy. Physical model tests are used to evaluate wave transmission past a near-shore floating WEC of the wave activated body type, named DEXA. Efficiency and transmission characteristics are approximated to functions of wave height, period and obliquity. Their order of magnitude are $20 \%$ and $80 \%$, respectively. It is imagined that an array of DEXA is deployed in front of Marina di Ravenna beach (IT), a highly touristic site of the Adriatic Coast. Based on the CERC formula, long-shore sediment transport is evaluated in presence and in absence of this array of DEXAs. The sediment transport in this site is quite large and frequently changes directions during the year. The larger North directed contribution and the more persistent South directed one are similar in magnitude and almost compensate each other, with the latter only slightly prevailing. It is shown that the DEXA could be designed so that the effect on sediment transport becomes quite significant and the direction of the net transport can be reversed.
\end{abstract}

Keywords: wave energy converters, wave transmission, efficiency, coastal defence, DEXA, experiments.

\section{INTRODUCTION}

Typical coastal defence schemes are based on a combination of artificial nourishment and different types of structures, such as submerged breakwaters, groins, artificial reefs, etc. The limit of these interventions is that their efficiency decreases with increasing water level.

As a result of climate change, the threat posed by coastal erosion and coastal flooding is driving toward the search for innovative protection schemes that are not affected by sea level rise.

Floating breakwaters, being almost independent from sea level rise, may be more suited to shelter the near-shore zone and reduce the maximum run-up in the long term. These structures partly reflect and partly dissipate the incident wave energy, so that the wave transmission is significantly reduced, at least for wave periods smaller than the design one.

The floating wave energy converters (WECs) are in principle even more suited to reduce transmission since they may add to the typical properties of floating breakwaters also the capacity of harvesting part of the incident wave energy. Several WECs have up to now been applied as demonstration and development projects but no application involving coastal protection has been proposed yet.

Aim of this note is to analyse the possible combined application of Wave Energy Converters (WECs) as a tools protect the coast and harvest energy.

WECs are intrinsically suited to severe environmental conditions, but the strategy associated to their survival might suggest that -under extreme storms- the power take off should be halted or operated in safe mode, thus reducing the additional capacity of energy extraction. This is necessarily associated to a lower energy absorption and, in final analysis, to a higher wave transmission. If the WEC is used also for erosion control purposes, it is not critical if the device is less effective during the most important storms, since erosion is a long term process integrating events in time. On the contrary, if the device is meant to protect against coastal flooding, the installation should be designed to operate also during rare and exceptional storms.

The specific WEC investigated in this note is named DEXA (www.dexawave.com). The DEXA belongs to the floating Wave Activated Body (WAB) type and the energy production is based on the relative movement of adjacent parts.

Preliminary tests have shown that, for high wave steepness, DEXA is very effective (Kofoed, 2009). This behaviour allows to design a device that produces energy also when the sea conditions are not extreme (i.e. when the waves are not particularly high) such as in case of strong winds in short fetches.

Wave transmission past WEC depends on the Power Take Off (PTO) load and on the incident wave characteristics (peak wave period $T_{\mathrm{p}}$ and significant wave height $H_{\mathrm{s}}$ ), on the geometrical and

\footnotetext{
${ }^{1}$ IMAGE, University of Padova, Via Ognissanti 39, 35129 Padova, Italy

2 DICAM, University of Bologna, Viale Risorgimento, 2, 40136 Bologna, Italy

${ }^{3}$ Department of Civil Engineering, University of Aalborg, Sohngaardsholmsvej 57, 9000 Aalborg, Denmark
} 
dynamic properties (mass, added mass, damping factor, natural period of oscillation, Martinelli et al, 2008) and finally on the characteristics of the mooring system (Giles M.L., R.M. Sorensen, 1979).

In order to assess if DEXA is a suitable solution for coastal defence, the following steps are carried out:

- analysis of the device efficiency $\eta$ and of the transmission coefficient $K_{\mathrm{T}}$; their variation with wave height, wave steepness, wave angle and water depth is obtained on the basis of experiments carried out in the deep water wave basin of Aalborg University. For given structure characteristics, the transmission coefficient $K_{\mathrm{T}}$ is mainly related to $l / L_{\mathrm{p}}$ being $L_{\mathrm{p}}$ the peak wave length and $l$ the breakwater length along the wave direction (in similitude to floating breakwaters; Hales, 1981). The larger the $l / L_{\mathrm{p}}$ value, the smaller the $K_{\mathrm{T}}$ coefficient. It is therefore expected that a sufficiently long DEXA device may be effective in reducing wave transmission;

- assessment of the near-shore morphological effects induced by a row of DEXA through an example application along the Adriatic coastline, Italy. By reducing wave transmission, the sediment transport must also be diminished, and the CERC formula is used to quantify these effects. In practice, the variation of the longshore net transport is evaluated by comparing the yearly volume with or without the device.

The paper gives at first a detailed description of the wave basin and of the device model, including the main geometrical properties, mooring system and measurement tools. The tested wave conditions are also provided. In the following section, the dependence of $K_{\mathrm{T}}$ on $l / L_{\mathrm{p}}$ is approximated by a curve fitted to the experimental results. Then, an hypothetical application of the device in front of the anticipated Northern Adriatic coast is presented to show how an appropriately designed device can even reverse the direction of the net sediment transport.

\section{EXPERIMENTAL INVESTIGATION}

An extensive investigation on the DEXA device is ongoing in the deepwater wave basin of Aalborg University. The purpose of the tests is to assess and improve the system efficiency and evaluate the reduction of wave agitation behind the model itself.

Kofoed (2009) and Zanuttigh at al. (2010) present part of the experiments. New tests carried out with oblique waves, that complete the database used for this paper, are described hereafter.

\section{The facility}

3D hydrodynamic tests are performed in the directional wave basin of the Hydraulics and Coastal Engineering Laboratory at Aalborg University, DK. The basin is $15.7 \mathrm{~m}$ long, $8.5 \mathrm{~m}$ wide and $1.5 \mathrm{~m}$ deep. The wave generator is a piston type paddle system composed of 10 actuators with stroke length of $0.5 \mathrm{~m}$, enabling generation of short-crested waves. The software used for controlling the paddle system to generate waves is AwaSys developed by the same laboratory. Regular and irregular longcrested waves with peak periods up to approximately 2.5 seconds, oblique $2 \mathrm{D}$ and $3 \mathrm{D}$ waves can be generated with good results.

The active absorption on the wave paddles is not used, but passive absorption is placed at the rear end of the basin and at both sides. The absorbing sidewalls are made of crates $(1.21 \times 1.21 \mathrm{~m}, 0.70 \mathrm{~m}$ deep). The 1:4 sloping beach placed opposite to the wave maker is made of concrete and gravel with $D_{50}=5 \mathrm{~cm}$.

\section{The DEXA model}

The DEXA device is developed and patented by DEXA Wave Energy ApS (www.dexawaveenergy.co.uk). The device (Fig. 1) is essentially formed by two catamarans placed side by side and hinged, so that that are allowed to pivot along their connection. The concept follows closely a famous design by Sir Christopher Cockerell ("Cockerell's raft"). The power take off system is based on Aquagear (i.e. a low pressure power transmission technology, based on water) and acts by restraining the angular oscillations. The two floaters of each catamaran are ideally distant $1 / 2$ wavelength. The dimensions of a device in prototype scale are $44 \mathrm{~m} \times 16.2 \mathrm{~m}$. According to a recent economical optimisation, the prototype scale DEXA placed in the North Sea should be rated $160 \mathrm{~kW}$. A device in scale 1:10 was temporarily deployed in the Lymfiord near Aalborg (Fig 2, left). A larger 1:5 scale model might be tested in the near future in Hanstholm Port and/or Nissum Bredning.

In the laboratory, DEXA is reproduced in 1:20 scale model (Fig. 2, right), resulting $2.10 \mathrm{~m}$ long and $0.81 \mathrm{~m}$ wide (perpendicularly to wave propagation), the total weight being $22 \mathrm{~kg}$. Two additional weights (formed by sand bags respectively $1.77 \mathrm{~kg}$ and $2.56 \mathrm{~kg}$ heavy) are placed over the model in 
order to reach the required draft. The measurement tools and the two added bags totally weight $10 \mathrm{~kg}$. The draft is such that at rest the free water surface passes in correspondence of the axis of the four buoyant cylinders.

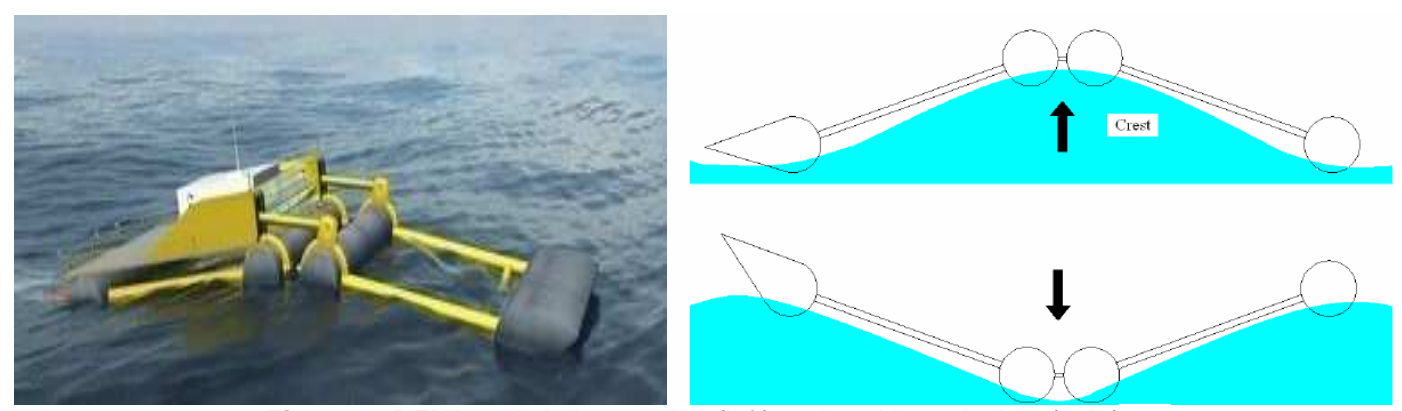

Figure 1: DEXA: artist's impression (left) and working principle (right).

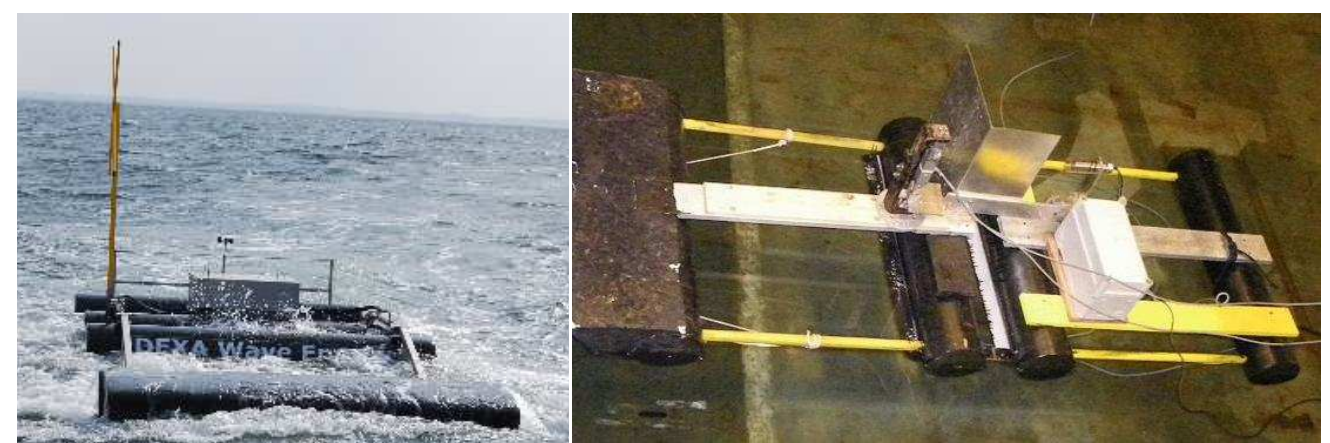

Figure 2: Model of DEXA deployed in Lymfiord (scale 1:10) and laboratory model (1:20 scale).

The Power Take-Off (PTO) will presumably consist of a low pressure transmission technology. The extracted power is essentially the moment multiplied by the angular velocity, where the moment is somewhat controlled by the generator to maximize the efficiency.

In order to simulate the effect of the PTO in a realistic way, a specific system was designed (Kofoed, 2009). The model of the PTO (see Figure 3), placed close to the centre of the system in order to maximise the stabilisation force, consists of a metal bar with an elongate-shaped hole, a wire welded at the two ends of the hole and a small electric engine with a wheel. The bar is connected to one half of the device and the wheel to the other, via a load cell, namely a strain gauge equipped "bone". The "bone", $10 \mathrm{~mm}$ thick, is placed on the PTO system and records the moment due to force exchanged between the pontoons.

The wire is rolled up around the wheel that is forced to rotate while translating along the bar hole. The load on the PTO is modified by varying the current in the engine and therefore the resistance of the wheel to rotation, so that the body rigidity is changed (in total it was possible to set up 17 rigidities).

Relative velocity is measured through a non-contact ultrasonic displacement sensor with a plate for the signal reflection.

\section{Test programme}

The measurements are carried out to determine the transmission coefficient $K_{T}$ and the produced power $P_{m}$.

Tested conditions differ with regard to the mooring system: single elastic spring, turret mooring and two types of spread moorings were analysed. Results used in this note derive from the two set of tests obtained with spread moorings, formed by four chains with compliant catenary configuration, fixed to the bottom with $20 \mathrm{~kg}$ gravity anchors Chains are connected to the DEXA in correspondence of the bar mid point, i.e. where the expected vertical oscillation is minimum. Chain weight was $1.1 \mathrm{~kg} / \mathrm{m}$, aiming at restraining the drift to approx $0.2 \mathrm{~m}$ in model scale ( $4 \mathrm{~m}$ in prototype). 


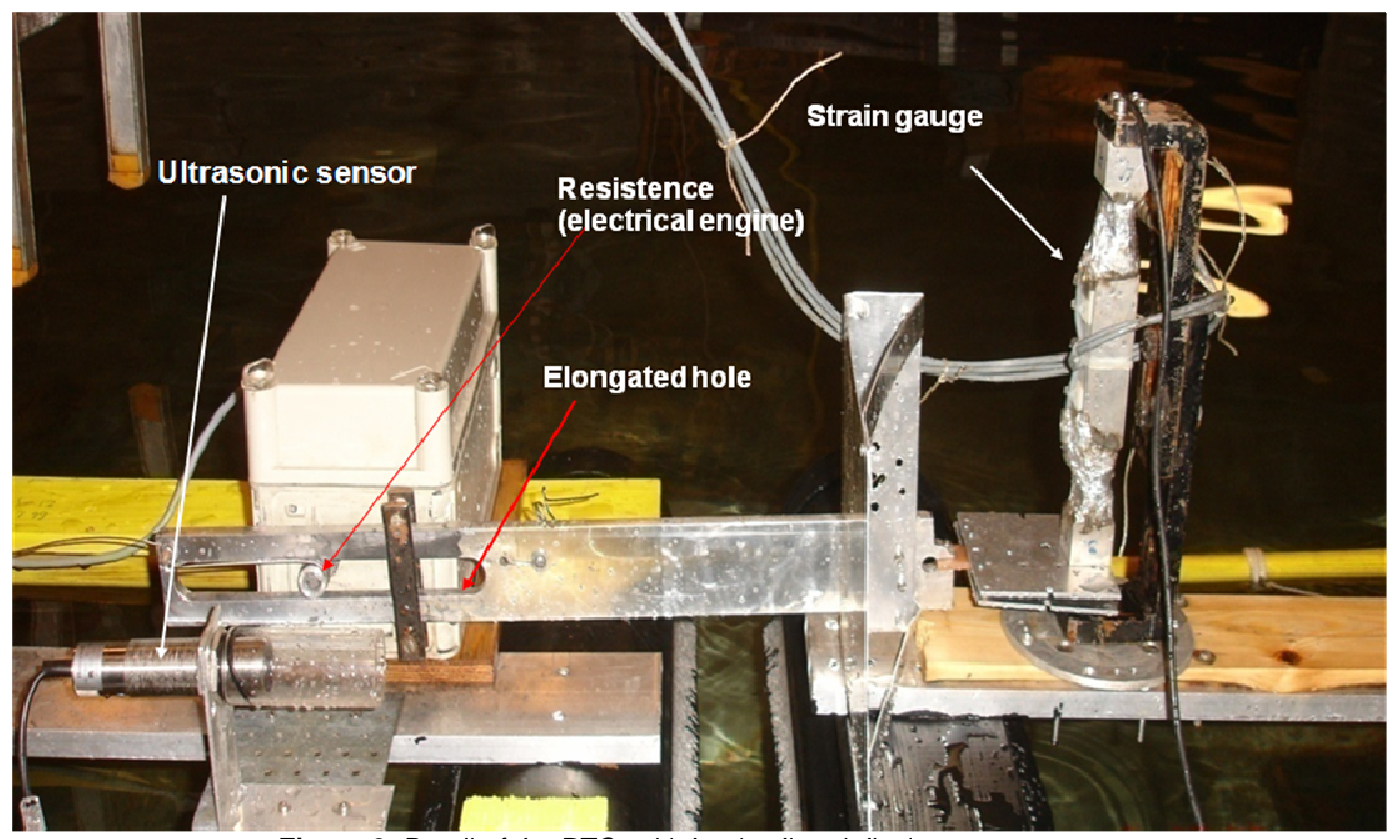

Figure 3: Detail of the PTO, with load cell and displacement meter.

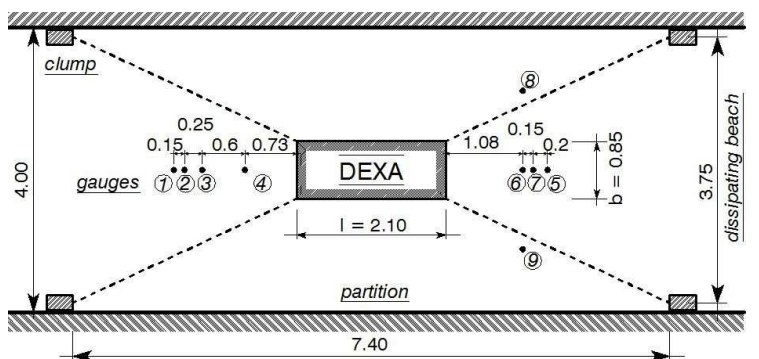

Figure 4: Test layout in wave basin ( $1^{\text {st }}$ set of tests: Zanuttigh et al, 2010).

The first set of tests are described in Zanuttigh et al, (2010), and only the essential results will be given in the following. The incident wave is always perpendicular and two water depth are examined $\left(h_{1}=0.55 \mathrm{~m}\right.$ and $\left.h_{2}=0.65 \mathrm{~m}\right)$. In order to represent arrays of devices distant $4 \mathrm{~m}$ to each other, a wall $0.75 \mathrm{~m}$ high is placed in the wave basin to limit its width. Figure 4 shows a plan view of the model including anchor layout. Nine resistive gauges are placed in the basin, four of which between the wave maker and the DEXA device and five between the device and the dissipating beach. The Mansard and Funke (1980) method is used to separate incident and reflected wave heights in front and behind the device, allowing to estimate the reflection and the transmission coefficients. Control gauges at the sides of the basin, placed at the distance of $1 \mathrm{~m}$ from the centreline, are used to evaluate the diffraction pattern behind the device.

Since the beach appeared to have fairly good absorbing properties, under irregular wave conditions the gauges 5, 6 and 7 did show the same value and such redundancy was eliminated on the following series of tests.

The second set of tests (Fig. 5) was specifically carried out to investigating the effect of wave obliquity. The model was rotated in the basin of $\theta_{1}=0^{\circ}, \theta_{2}=15^{\circ}$ and $\theta_{3}=30^{\circ}$. Two plates were placed $1 \mathrm{~m}$ off the centreline to limit wave diffraction in this region.

In order to allow weathervaning, a compliant spread mooring system was used, where all chains point to a single point, placed forward of the centre of rotation of the device (Fig. 5). Therefore in presence of oblique wave attack, the DEXA rotates toward the incoming waves.

The chain weight was rather high, $1.1 \mathrm{~kg} / \mathrm{m}$, to ensure a compliant response under extreme conditions in absence of one of the front chains. Unfortunately this increased the device stiffness to yaw so that the weathervaning of the device was only $70 \%$ of the total for high wave conditions $\left(11.5^{\circ}\right.$ rotation for $15^{\circ}$ obliquity, $17^{\circ}$ rotation for $30^{\circ}$ obliquity, $H \mathrm{~s}=17.5 \mathrm{~cm}, T \mathrm{p}=2.5 \mathrm{~s}$ ).

Details on the configurations for these second set of tests may be found in Boscolo (2010). 


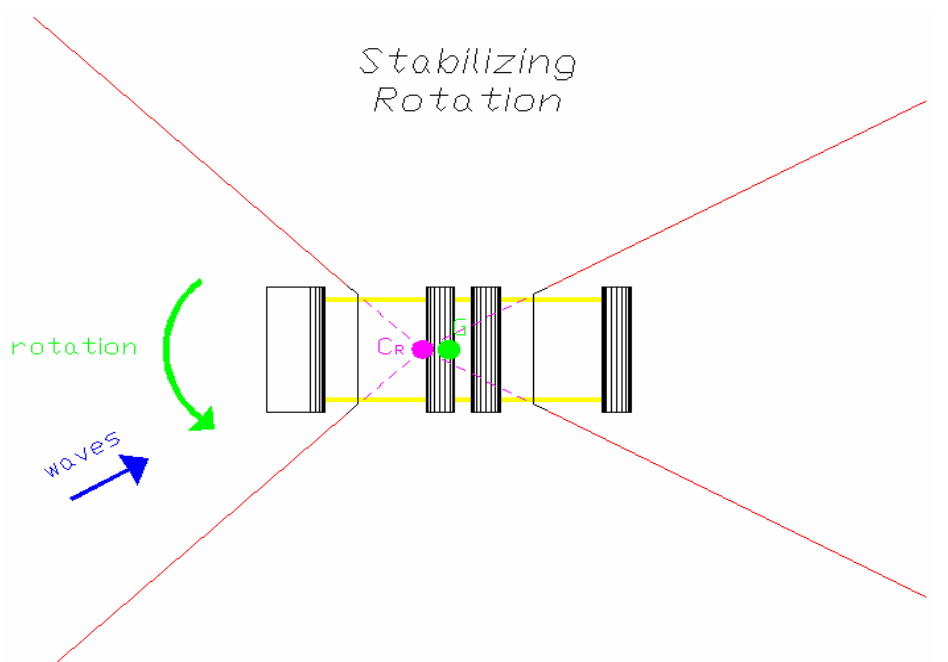

Figure 5: Test layout in wave basin ( $2^{\text {nd }}$ set of tests: Boscolo, 2010).

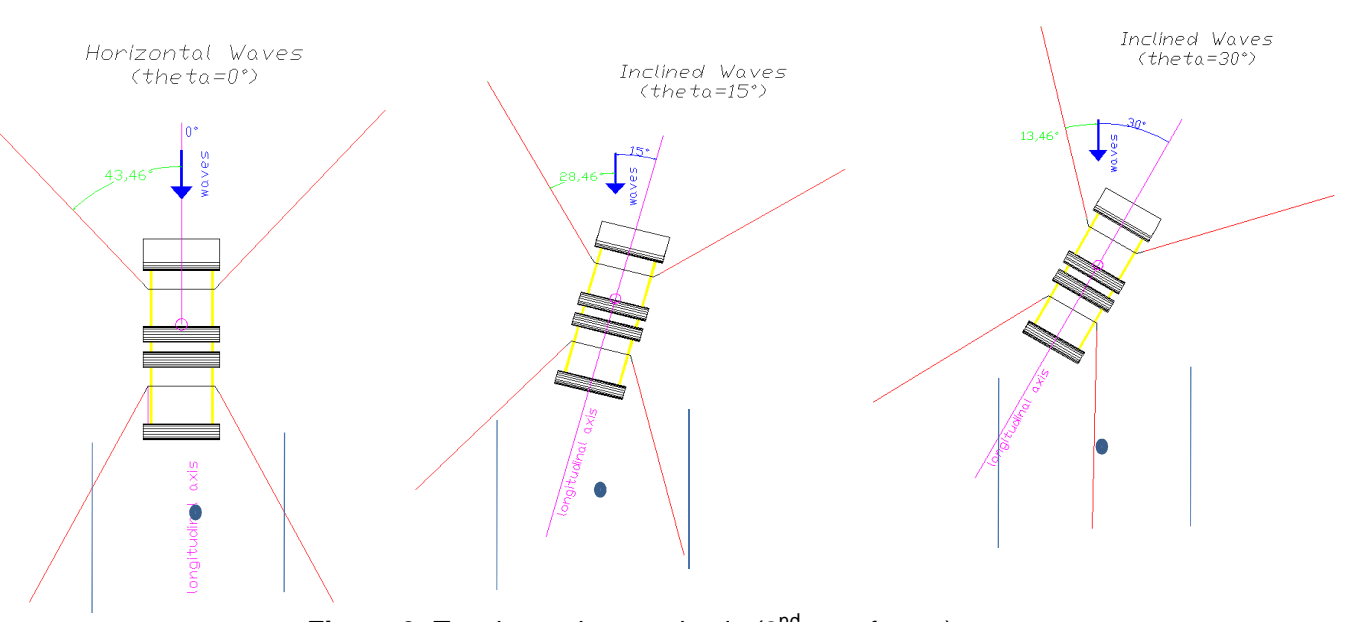

Figure 6: Test layout in wave basin $\left(2^{\text {nd }}\right.$ set of tests $)$.

\section{Wave conditions}

Regular and irregular waves were tested. The first are merely used to define the optimal PTO rigidity $R$ (a variable resistance assuming values in the range 0-17, with higher values for higher loads), i.e. the condition that maximises the power $P_{m}$. Regular waves are used in this case since the duration of each wave attack is much lower (5 minutes) than for irregular tests (20 minutes).

Irregular waves are characterised by $\mathrm{Hs}$ in the range $0.05-0.25 \mathrm{~cm}$ and wave steepness in the range 15\%. The complete list of tested waves is given in Zanuttigh et al, 2010.

\section{TEST RESULTS}

\subsection{Device efficiency $\eta$}

The device efficiency $\eta$ is defined as the capture width divided by the device width:

$$
\eta=\frac{P_{m}}{P_{w} \cdot b}
$$

It is a synthetic parameter that basically provides an overview of the working configurations. 
Figure 7 shows the measured values of $\eta$ as a function of $l / L_{p}$. From this Figure, it is possible to notice that in order to improve the performances of DEXA (i.e. maximize the efficiency $\eta$ ), the dimensionless length of the model should be close to 1 .

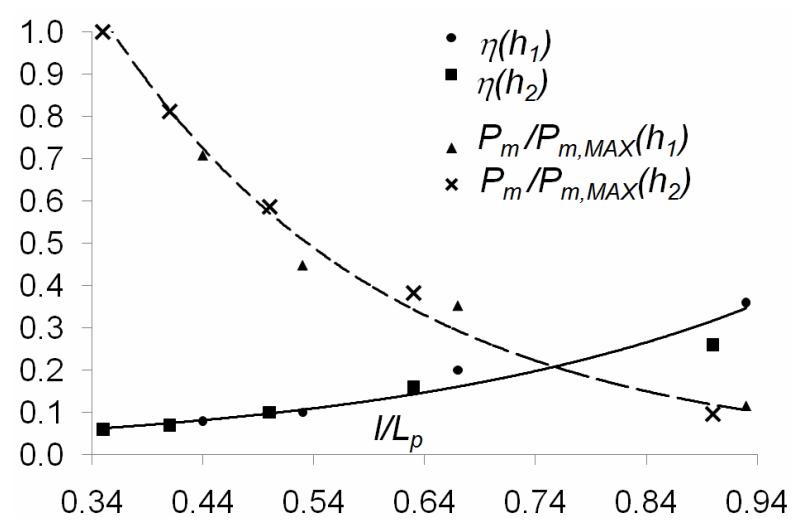

Figure 7: Variation of $\eta$ and $P_{m} / P_{m, M A X}$ vs $l / L$

Experimental values of both $\eta$ and $P_{m}$ in Figure 7 can be fitted by exponential tendency curves whose coefficients are computed by means of the ordinary least squares method:

$$
\begin{aligned}
& \eta(l / L)=0.022 \exp [2.95 \cdot l / L] \\
& P_{m}(l / L)=10.7 \exp [-3.94 \cdot l / L]
\end{aligned}
$$

The coefficient of correlation is 0.96 for $\eta$ and 0.98 for $P_{m}$. It can be noticed that with increasing $l / L_{p}$ the data scatter increases, especially for $\eta$.

It was also observed that $\eta$ gradually increases with increasing wave steepness (Zanuttigh et al, 2010), confirming that DEXA is particularly effective for waves in near breaking conditions.

Based on the occurrence of each wave condition the yearly power $P_{Y}$ at full scale is $214.5 \mathrm{MWh} / \mathrm{y}$ if the device is placed at $h_{2}$ and $178.3 \mathrm{MWh} / \mathrm{y}$ at $h_{1}$. Although it seems more convenient to install the device in deep waters, the larger power production $P_{Y}$ has to cope with the installation and maintenance costs, that generally are larger than in lower depths.

\subsection{Transmission coefficient $K_{T}$}

The variation of $K_{T}$ is summarised in Figure 8, showing a clear dependence on wave length $L_{p}$, as it can be expected from floating devices (Martinelli et al, 2008). Specifically if $l / L_{p}$ increases, $K_{T}$ decreases and the decreasing rate of $K_{T}$ is larger on deeper bottoms. In fact for a fixed value of $l / L_{p}, K_{T}$ is lower for $h_{2}$ rather than for $h_{l}$.

Experimental results may be approximated by a linear regression both for $h_{l}$ and $h_{2}$ water depths:

$$
K_{T}(l / L)=1.04-0.234 \cdot l / L
$$

when $0.30<l / L_{p}<0.94$. The correlation coefficient in this case is quite low, 0.70 .

The measured values of KT $(>0.77)$ for a single device show that the reduction of wave energy obtained with one row of DEXA is certainly lower than the reduction usually obtained with a submerged coastal structure. The still high residual wave agitation suggests that it would be therefore reasonable to place more than one row of devices to reduce KT and at the same time increase wave energy production. 


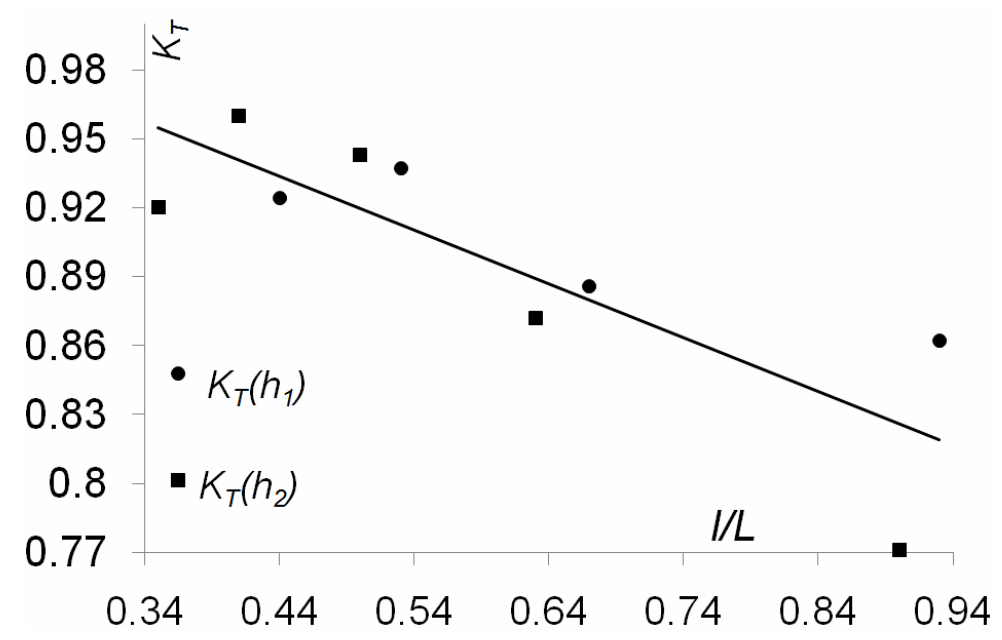

Figure 8: Variation of transmission coefficient $K_{T}$ with $l / L_{p}$

Figure 9 presents the dependence of $K_{T}$ on wave steepness $s$.

The dependence of $K_{\mathrm{T}}$ on $s$ is indeed weak especially when $l / L_{p}$ is quite low $\left(l / L_{p}<0.6\right)$. For a fixed value of $l / L_{p}, K_{\mathrm{T}}$ decreases and at the same time (see Table 4) $\eta$ increases with increasing $s$.

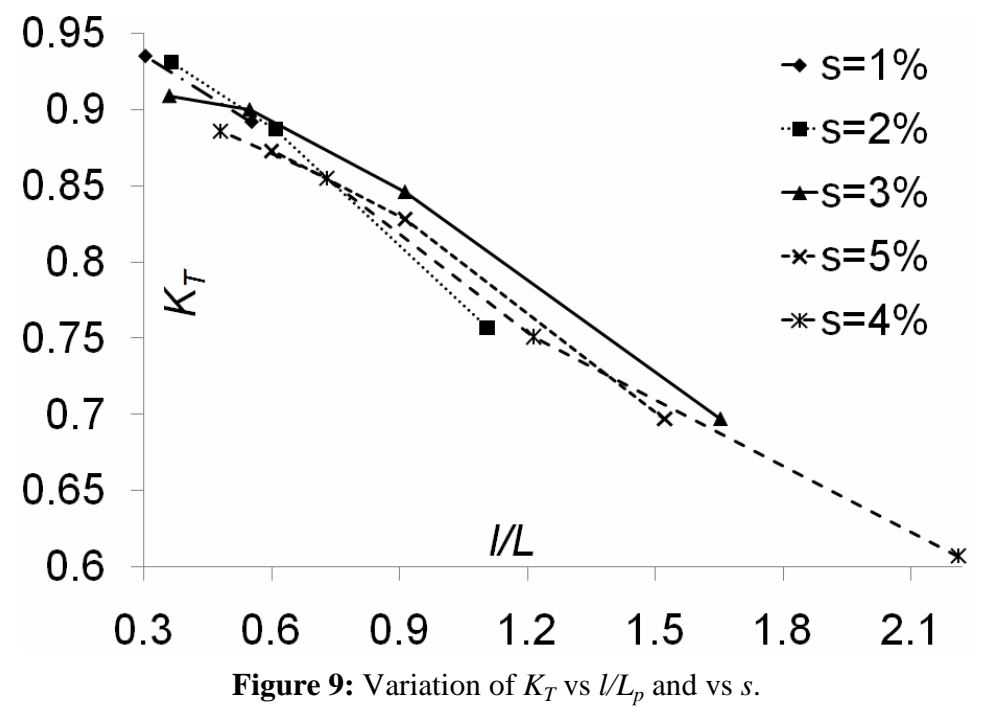

The effect of wave obliquity can be observed in Figure 10, showing the results relative to the second set of tests. There is certainly a reduction of wave transmission with increasing obliquity, and the same trend is maintained with respect to wavelength.

The following model is therefore proposed, that can be considered a correction factor to Eq. 4:

$K_{T}(l / L, \vartheta)=\min \left(0.3, K_{T}(l / L) K_{T}(\vartheta)\right)$

$K_{T}(\vartheta)=\cos (\vartheta)^{0.5}$

Fig. 11 shows the model for $K_{\mathrm{T}}$ and agreement of the fitting. 

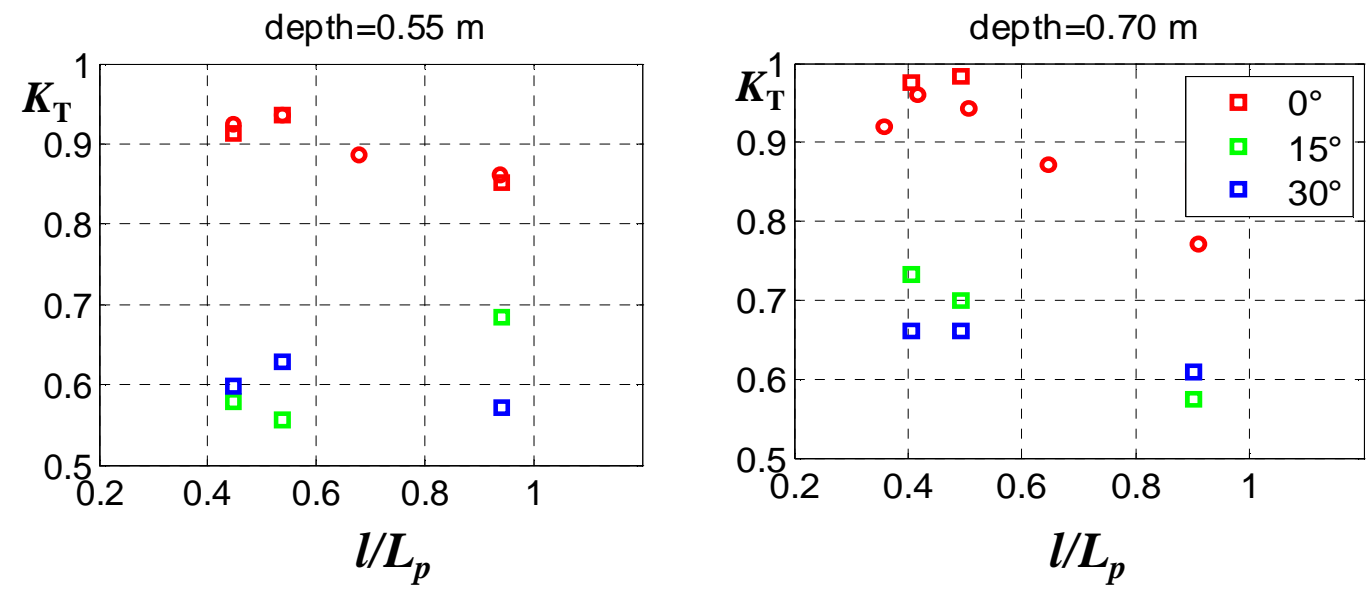

Figure 10: Variation of wave transmission with depth, obliquity and wavelength. It can be noticed that for oblique waves the transmission decreases. Red circles are related to the first set of tests, i.e. with $0^{\circ}$, relative to depth of 0.55 and $0.65 \mathrm{~m}$ (whereas the second set was tested for water depth 0.55 and $0.7 \mathrm{~m}$ ).
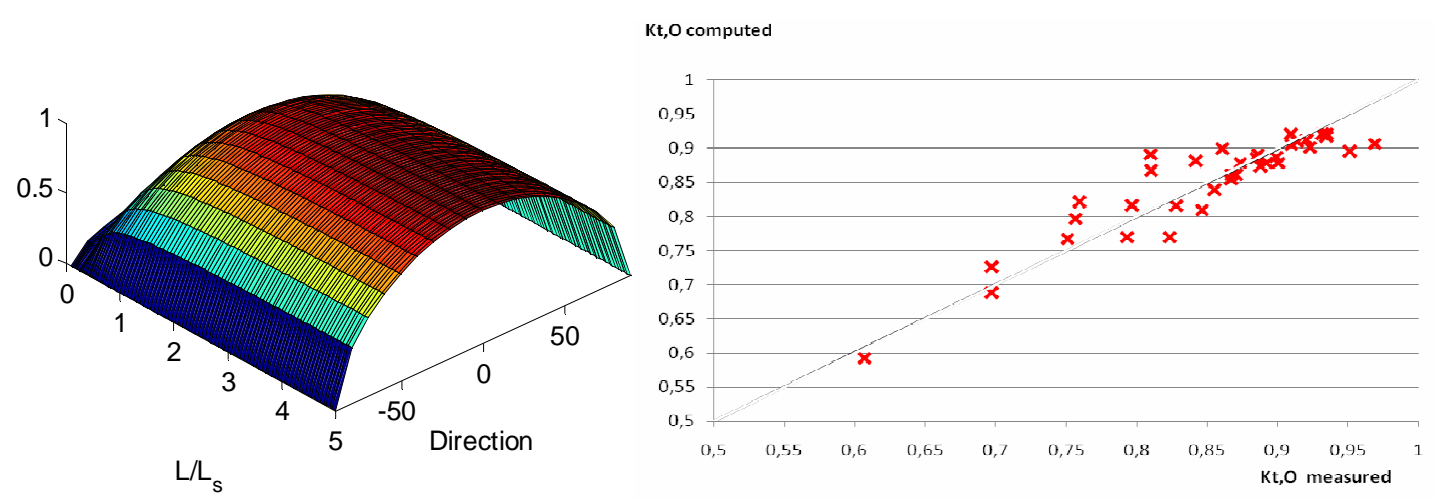

Figure 11: Left: modelled transmission coefficient (without lower limit); right agreement between measured and predicted values of transmission coefficient (Eq. 4, 5)

\section{EXAMPLE APPLICATION}

In this section, a simple example is proposed to investigate on the possible advantages of a park of WECs in terms of coastal protection.

In particular, sediment transport for Milano Marittima beach is evaluated using the CERC formula, in presence and in absence of a line of DEXA.

The site of Milano Marittima, Ravenna, is a famous touristic resort between Lido di Savio and Cervia. The coastline is maintained by a periodic nourishment of order $20^{\prime} 000 \mathrm{~m}^{3} / \mathrm{y}$, compensating the erosive trend that is mainly due to natural subsidence. In the area, a southward directed sediment transport accumulates $10^{\prime} 000 \mathrm{~m}^{3} / \mathrm{y}$ toward the port of Cervia. In Martinelli at al, 2007, recent works combining port dredging at Cervia and nourishment with submarine sands on the northern beach are described.

Figure 12 shows the site and the wave climate in point $44^{\circ} \mathrm{N}, 13^{\circ} \mathrm{E}$, placed off-shore the site under analysis according to MEDATLAS (Medatlas Group, 2004) tables of wave height and direction (based on forecasted winds).

The characteristic of the site is that waves from North (Bora winds) are steeper, whereas waves from the South are longer (Scirocco). Therefore a constant wave steepness of 0.04 and 0.02 are assumed for the respective conditions.

The array of DEXA is tentatively formed by a line of devices spaced as in the model, at a scale 8 times larger, i.e. one device $6.8 \mathrm{~m}$ wide every $32 \mathrm{~m}$, on a water depth of $6 \mathrm{~m}$ (corresponding to $630 \mathrm{~m}$ offshore).

The CERC formula based of such climate is used to evaluate the average longshore sediment transport. 


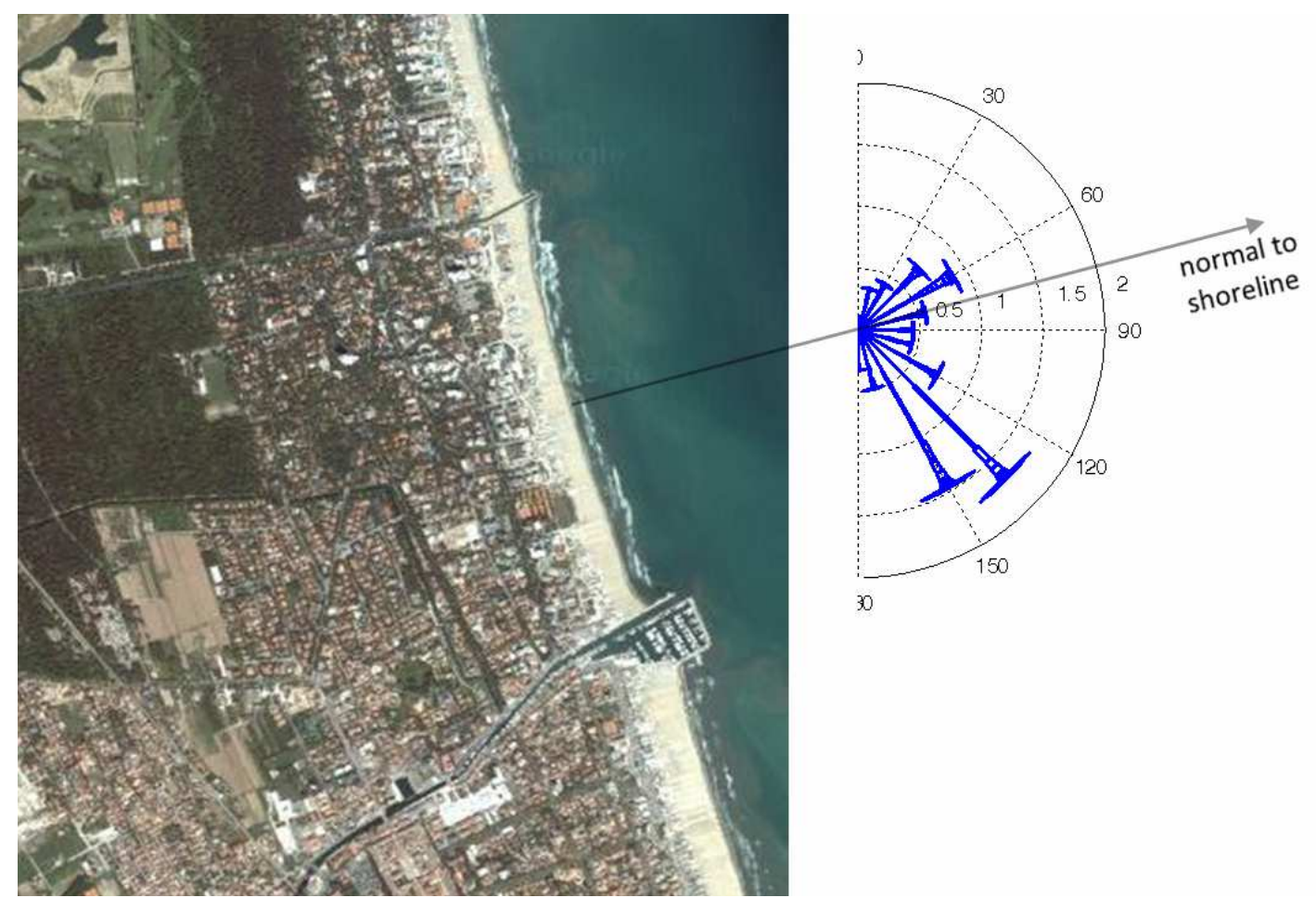

Figure 12: The site and the wave climate (source Med Atlas, 44N, 13E)

The following assumptions adopted in the computation:

- linear shoaling and refraction until breaking point, with breaking index $\gamma_{\mathrm{b}}=0.78$;

- sediment transport evaluated for each single wave attack characterized by $H_{\mathrm{s}}, T_{\mathrm{p}}$ and $\theta$, is multiplied by the frequency of occurrence regardless of the actual sequences of events;

- a tri-angular distribution of sediment transport along the cross-shore section is assumed varying from "zero" at shoreline and at the depth of closure $h_{\mathrm{c}}$, and with maximum at the breaking point; depth of closure is evaluated per each wave attack as $h_{\mathrm{c}}=2 H_{\mathrm{s}}$ (Hanson, 1989);

- the DEXA length $l$ is $16.8 \mathrm{~m}$; this dimension is used in Eq. 4, 5 to compute the wave height incident onshore.

The obtained total sediment transport is southward directed and equal to $200^{\prime} 000 \mathrm{~m}^{3} / \mathrm{y}$. Accuracy of the result is not of great concern of this investigation, since the simplified computation aims at evaluating the relative importance of the presence of WECs. Nevertheless, the result agrees with the value suggested by the Coastal Plan of Emilia Romagna, 1996.

The computed distribution across the profile is given in Fig. 13. It can be seen that the net transport is the difference between two large values directed in opposite directions. The net transport is positive (northward directed) only close to the shore, but in total it is negative (southward directed).

Figure 14 compares the sediment transport distribution given in Figure 13 to the one obtained in presence of the DEXA. It can be seen that, as a consequence of the reduction of the wave height, the sediment transport is also reduced. It is interesting to notice that the reduction of the two components (North and South directed) is not proportional, and the distribution of the net transport in presence and in absence of the WEC is quite different: in presence of the DEXA, the maximum of the curve is less than half in amplitude and it is placed more southward.

Figure 15 shows the result of a peculiar use of the device. It is assumed that the DEXA is automatically controlled and turns off the PTO when the waves are coming from the south. As a consequence, the DEXA floats freely and the apparent length of the body is half of the full one. The DEXA merely rides on the waves, and therefore its capability of extracting the incident wave energy is deleted. As a consequence, wave transmission changes. It is assumed in the computations, as a mere example, that transmission is 1 when waves are North directed. It is interesting to observe that such strategy reverses the net transport, with many evident practical implications. 


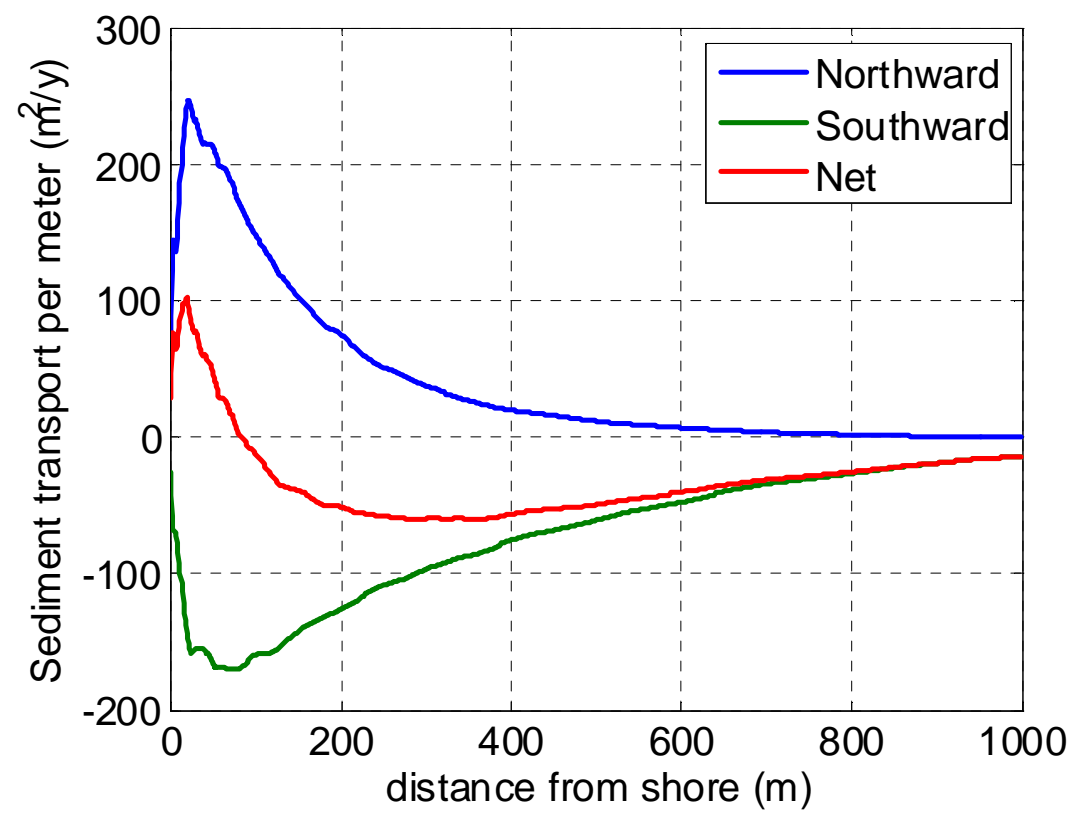

Figure 13: Distribution of sediment transport computed for Milano Marittima. The sediment transport is southward directed, except for a small region close to shore. A north-directed contribution on the first $50 \mathrm{~m}$ form shore is visible.

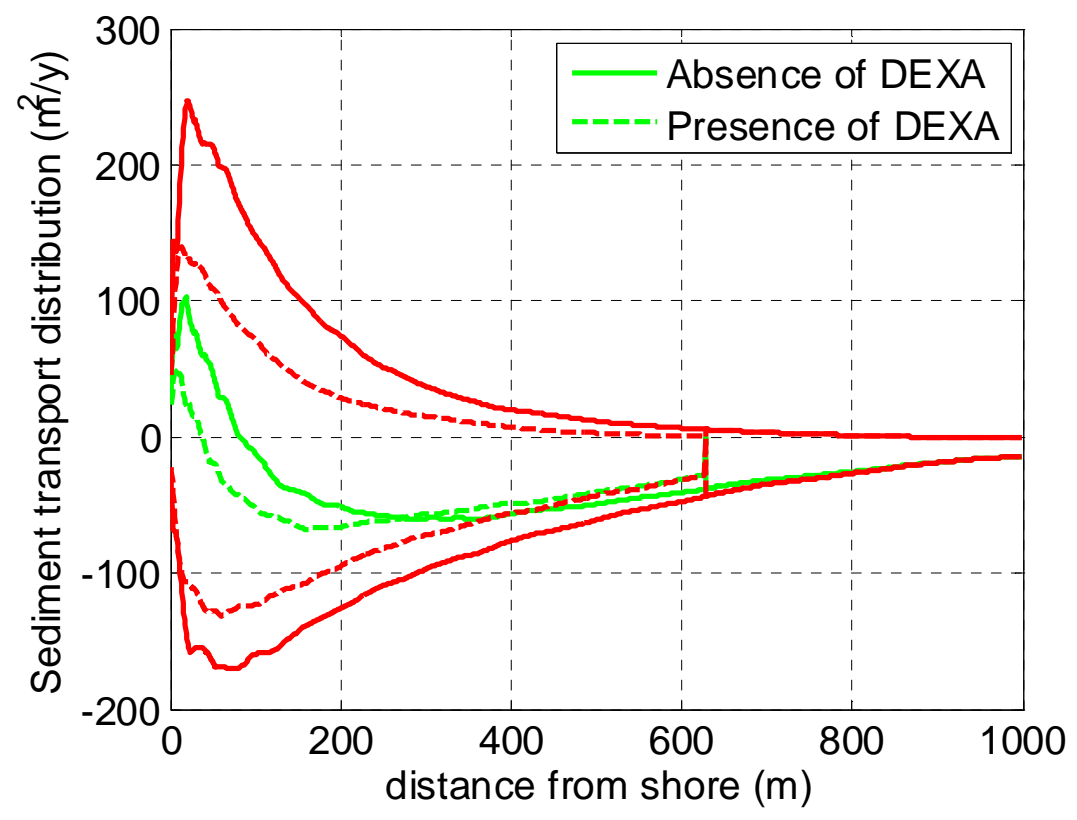

Figure 14: Distribution of sediment transport in absence and in presence of an array of DEXA $630 \mathrm{~m}$ from shore. In the latter case, the wave is reduced according to Eq. 4,5. The north-directed contribution on the first $50 \mathrm{~m}$ form shore is much reduced.

\section{Interpretation of the results}

The CERC formula suggests a dependency of sediment transport on wave height with power law 2.5. If we assume that the wave transmission behind the WEC is $K_{\mathrm{T}}$ of order 0.8 (when $l \approx L_{\mathrm{p}}$ ), this corresponds to a decrease in sediment transport of $43 \%$.

The reduction is significant but it is not equal for all waves. Longer waves, associated to higher transport, are less reduced. 


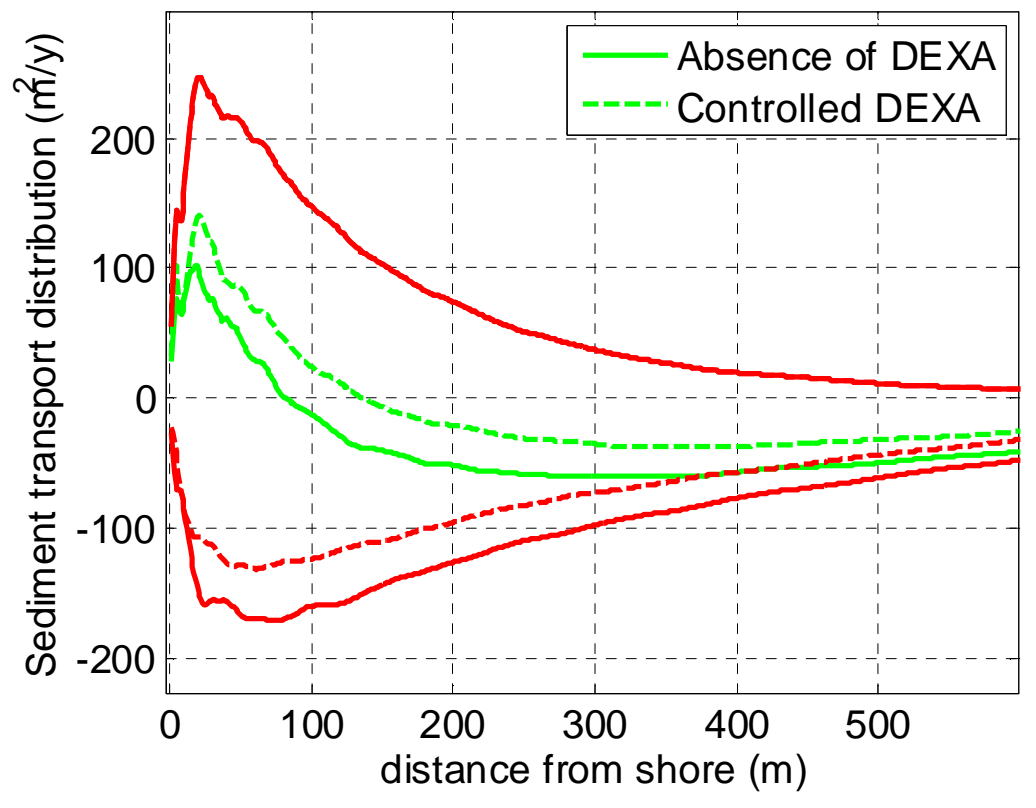

Figure 15: Distribution of sediment transport in case the DEXA is actively activated. Only when wave are coming from north, the DEXA is activated and wave is reduced. When waves come from the north, the DEXA is shut down and rides the waves without affecting them.

According to the example case, it can be concluded that if the longshore sediment transport is characterized by two opposite directions during the year, the WEC can operate selectively only on one single direction of the sediment transport and effectively reduce the net transport or even reverse its direction.

In order to better understand this concept, an simple wave climate is considered in Fig. 16, formed by just two waves, a smaller and more steep wave from North, a higher, longer and more frequent wave from South: $H_{\mathrm{s}}=2.0, s_{\mathrm{op}}=4.5 \%, \theta=-30^{\circ}, \mathrm{p}=10 \%, H_{\mathrm{s}}=2.5, s_{\mathrm{op}}=2.5 \%, \theta=+30^{\circ}, \mathrm{p}=17 \%$.
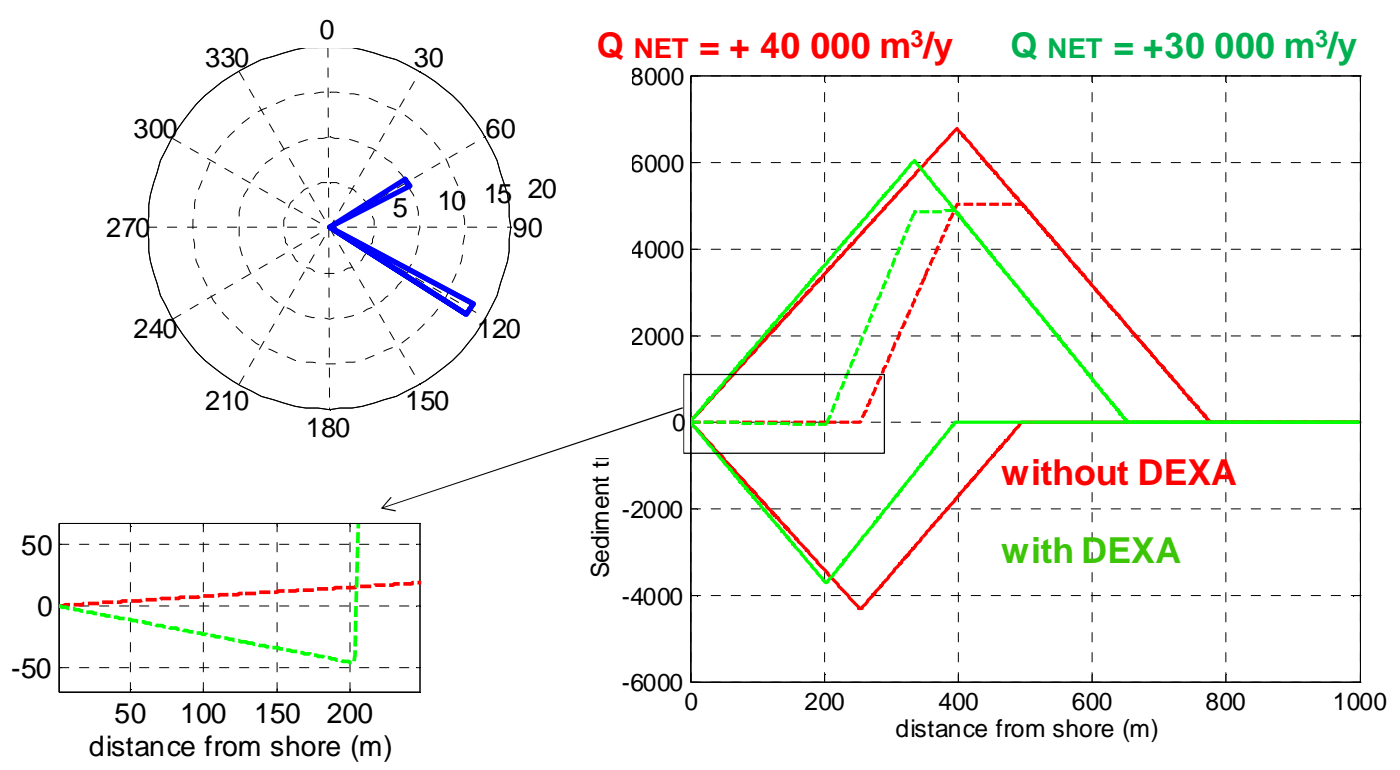

Figure 16: Distribution of sediment transport for a simplified wave climate. 
Each wave case, according to the hypotheses, is responsible of two triangular distributions of the sediment transport, respectively southward and northward directed. The net transport (dotted line) has a distribution shifted in space. In this case, there is even an inversion on the direction of the transport in proximity of the shoreline.

\section{CONCLUSIONS}

Conclusions are based on two set of physical model tests carried out in Aalborg deepwater wave basin on the DEXA device.

Energy efficiency is in between $10 \%$ and 35\% (maximum for devices having length equal to the wavelength).

Wave transmission $\left(K_{\mathrm{T}}\right)$, evaluated both for perpendicular and oblique wave attacks, was found in the range $0.6-0.9$ and is larger for longer structures. $K_{\mathrm{T}}$ was expressed as function of peak wave period and obliquity (wave height was considered less influent).

By an example application, a park of WECs is seen to reduce the wave energy along the coast and consequently the sediment transport; it was shown that the modest effect on wave attenuation can have a more evident effect on the net sediment transport process.

\section{ACKNOWLEDGMENTS}

The support of the EU Collaborative Project Theseus "Innovative technologies for safer European coasts in a changing climate", contract ENV.2009-1, ${ }^{\circ} 244104$, is gratefully acknowledged.

\section{REFERENCES}

Giles M.L., R.M. Sorensen (1979). Determination of mooring loads and transmission for a floating tire breakwater. Proc. Coastal Structures 1979, vol II. ASCE, 1069-1086.

Hales L.Z. (1981). Floating breakwaters: State-of-the-art literature review. US Army Coastal Engineering Research Center. CERC-TR-81-1.

Hanson H. (1989): GENESIS: a generalized shoreline change numerical model, J. of Coastal Res., 5(1), $1-27$.

Kofoed, J. P (2009). Hydraulic evaluation of the DEXA wave energy converter. DCE Contract Report No. 57. Dep. of Civil Eng., Aalborg University, Apr. 2009.

Mansard E.P.D, E.R. Funke (1980). The measurement of incident and reflected spectra using a least squares method. Proc. of the 17th Int. Conf. on Coastal Engineering, ASCE.

Martinelli L., P. Ruol, B. Zanuttigh, (2008). Wave basin experiments on floating breakwaters with different layouts, Applied Ocean Research, 30, 199-207.

Martinelli L., R. Durán, M. Nuez, B. Alonso, B. Zanuttigh, A. Lamberti (2008): Management of sedimnent stocks at El Masnou marina and Cervia Port, electronic version, Proc. Littoral 2008, $6 \mathrm{pp}$.

Medatlas Group, (2004): Wind and Wave Atlas for the Mediterranean Sea, W.E.A.O. Research Cell.

Shore protection manual, (1984). 4th ed., 2 Vol, U.S. Army Engineer Waterways Experiment Station, U.S. Government Printing Office, Washington, DC.

Zanuttigh B., L.Martinelli, M.Castagnetti, P.Ruol, J.P.Kofoed, P.Frigaard (2010): Integration of wave energy converters into coastal protection schemes, Proc ICOE 2010, 6 pp.

\section{APPENDIX - NOTATION}

\section{$b \quad$ model width}

$l \quad$ model length

$H_{s} \quad$ significant wave height

$H_{I} \quad$ significant incident wave height

$H_{T} \quad$ significant transmitted wave height

$T_{p} \quad$ peak period

$T_{s} \quad$ significant period

$s \quad$ peak wave steepness
$L_{p} \quad$ peak wave length

$l / L_{p} \quad$ dimensionless model length

$R \quad$ PTO rigidity

$P_{w} \quad$ incident wave power

$P_{m} \quad$ produced mechanical power

$\eta \quad$ efficiency of the device

$K_{T} \quad$ transmission coefficient 